\title{
Novel Heterocycle-Based Two-Photon Absorbing Dyes
}

Alessandro Abbotto, Luca Beverina, Renato Bozio, Antonio Facchetti, Camilla Ferrante, Giorgio

A. Pagani, Danilo Pedron, and Raffaella Signorini

\section{Supporting Information}

\section{Experimental}

${ }^{1} \mathrm{H}$ and ${ }^{13} \mathrm{C}$ NMR spectra were recorded using a Bruker AMX-500 spectrometer operating at 500 and $125.70 \mathrm{MHz}$, respectively. The spectral parameters and calibrations have been previously reported. ${ }^{1}$ Coupling constants are presented in Hertz. Anhydrous $N, N$-dimethylformamide (DMF) was supplied by Fluka and stored over molecular sieves. Anhydrous acetonitrile was supplied by Aldrich and stored under nitrogen. Melting points are uncorrected.

1-(N-Methylpyrid-4-yl)-2-(5-formyl- $N$-methylpyrrol-2-yl)ethene triflate (4). Freshly distilled $\mathrm{POCl}_{3}(0.449 \mathrm{~g}, 2.93 \mathrm{mmol})$ was added dropwise to anhyd DMF (0.214 g, $\left.2.93 \mathrm{mmol}\right)$ under nitrogen at $5{ }^{\circ} \mathrm{C}$. A solution of 1-(N-methylpyrid-4-yl)-2-( $N$-methylpyrrol-2-yl)ethylene $\operatorname{triflate}^{2}(0.700 \mathrm{~g}, 2.01 \mathrm{mmol})$ in anhyd acetonitrile $(15 \mathrm{~mL})$ was added dropwise to the reaction mixture at $5{ }^{\circ} \mathrm{C}$. After the mixture was stirred for $4 \mathrm{~h}$ at room temperature, the formed precipitate was filtered off under reduced pressure and washed with aq $\mathrm{K}_{2} \mathrm{CO}_{3}(5 \mathrm{~mL})$, to give the product as a yellow solid $(0.530 \mathrm{~g}, 1,41 \mathrm{mmol}, 70 \%): \mathrm{mp}=189-191{ }^{\circ} \mathrm{C}\left(\mathrm{H}_{2} \mathrm{O}\right) ;{ }^{1} \mathrm{H}$ NMR $\left(\right.$ DMSO- $\left.d_{6}\right) \quad 9.63(\mathrm{~s}$, $1 \mathrm{H}), 8.85(\mathrm{~d}, 2 \mathrm{H}, J=6.7), 8.27(\mathrm{~d}, 2 \mathrm{H}, J=6.8), 8.01(\mathrm{~d}, 1 \mathrm{H}, J=16.1), 7.50(\mathrm{~d}, 1 \mathrm{H}, J=16.1), 7.14$ $(\mathrm{d}, 1 \mathrm{H}, J=4.4), 6.99(\mathrm{~d}, 1 \mathrm{H}, J=4.4), 4.24(\mathrm{~s}, 3 \mathrm{H}), 4.08(\mathrm{~s}, 3 \mathrm{H}) ;{ }^{13} \mathrm{C}$ NMR $\left(\mathrm{DMSO}-d_{6}\right) 180.0,151.2$, 145.0, 140.0, 135.0, 127.0, 125.0, 123.5, 111.0, 48.5, 31.5. Anal. Calcd for $\mathrm{C}_{15} \mathrm{H}_{15} \mathrm{~F}_{3} \mathrm{~N}_{2} \mathrm{O}_{4} \mathrm{~S}: \mathrm{C}, 47.87$; H, 4.02; N, 7.44. Found: C, 47,68; H, 4.21; N, 7.80. 


\section{2,5-Bis[1-(4- $N$-methylpyridinium)ethen-2-yl)]- $N$-methylpyrrole ditriflate (1). Methyl}

triflate $(2.87 \mathrm{~g}, 17.5 \mathrm{mmol})$ was added dropwise to a solution of 4-picoline (1.63 g, $17.5 \mathrm{mmol})$ in dry benzene $(18 \mathrm{~mL})$ at room temperature under dry atmosphere. After stirring for $20 \mathrm{~h}$ the precipitate was isolated to give practically pure $N$-methyl-4-picolinium triflate as a white solid (4.38 g, $17.0 \mathrm{mmol}, 97 \%): \mathrm{mp} 110-11{ }^{\circ} \mathrm{C} ;{ }^{1} \mathrm{H}$ NMR (DMSO- $\left.d_{6}\right) \delta 8.82(\mathrm{~d}, 2 \mathrm{H}, J=7.1), 7.94(\mathrm{~d}, 2 \mathrm{H}, J=$ 7.1), 4.26 (s, 3H), 2.59 (s, 3H). A solution of $N$-methyl-4-picolinium triflate $(0.424 \mathrm{~g}, 1.65 \mathrm{mmol})$ in $\mathrm{EtOH}(8 \mathrm{~mL})$ and a few drops of piperidine were added to a solution of $4(0.621 \mathrm{~g}, 1.65 \mathrm{mmol})$ in the same solvent $(12 \mathrm{~mL})$. The reaction mixture was refluxed and then cooled to room temperature. The formed precipitate was filtered off under reduced pressure and washed with absolute EtOH (4 mL) to give the product as a dark solid (0.680 g, $1.13 \mathrm{mmol}, 68 \%): \mathrm{mp} \mathrm{300-304}$ ${ }^{\circ} \mathrm{C}\left(\mathrm{H}_{2} \mathrm{O}\right) ;{ }^{1} \mathrm{H}$ NMR (DMSO- $\left.d_{6}\right) \quad 8.66(\mathrm{~d}, 4 \mathrm{H}, J=6.8), 8.08(\mathrm{~d}, 4 \mathrm{H}, J=6.9), 7.91(\mathrm{~d}, 2 \mathrm{H}, J=15.9)$, $7.20(\mathrm{~d}, 2 \mathrm{H}, J=15.9), 7.02(\mathrm{~s}, 2 \mathrm{H}), 4.15(\mathrm{~s}, 6 \mathrm{H}), 3.80(\mathrm{~s}, 3 \mathrm{H}) ;{ }^{13} \mathrm{C}$ NMR (DMSO- $\left.d_{6}\right) \quad$ 152.5, 144.5, 135.6, 128.0, 121.2, 122.7, 113.5, 46.5, 30.8. Anal. Calcd for $\mathrm{C}_{23} \mathrm{H}_{23} \mathrm{~N}_{3} \mathrm{~F}_{6} \mathrm{~S}_{2} \mathrm{O}_{6}: \mathrm{C}, 44.88 ; \mathrm{H}, 3.77 ; \mathrm{N}$, 6.83. Found: C, 44.24; H, 3.77; N, 6.41.

1-(N-Methylquinol-4-yl)-2-( $N$-methylpyrrol-2-yl)ethene triflate (5). A solution of 1,4dimethylquinolinium triflate ${ }^{3}(1.00 \mathrm{~g}, 3.3 \mathrm{mmol})$ and piperidine $(0.1 \mathrm{~mL})$ in EtOH $(10 \mathrm{~mL})$ was added to a solution of $\mathrm{N}$-methyl-2-pyrrolecarboxaldehyde $(0.371 \mathrm{~g}, 3.4 \mathrm{mmol})$ in the same solvent $(10 \mathrm{~mL})$ at room temperature. The reaction mixture was stirred under reflux for $2 \mathrm{~h}$ and then cooled in an ice bath. A bright violet precipitate was separated by filtration and washed with ethanol (3 $\mathrm{mL})$ to give the almost pure product as a violet solid $(0.850 \mathrm{~g}, 2.15 \mathrm{mmol}, 65 \%): \mathrm{mp} 215-217{ }^{\circ} \mathrm{C}$; ${ }^{1} \mathrm{H}$ NMR (DMSO- $\left.d_{6}\right) \quad 9.10(\mathrm{~d}, 1 \mathrm{H}, J=6.7), 8.95(\mathrm{~d}, 1 \mathrm{H}, J=8.5), 8.46(\mathrm{~d}, 1 \mathrm{H}, J=8.8), 8.34(\mathrm{~d}, 1 \mathrm{H}$, $J=6.7), 8.20(\mathrm{t}, 1 \mathrm{H}, J=7.5), 8.14(\mathrm{~d}, 1 \mathrm{H}, J=15.5), 7.97(\mathrm{t}, 1 \mathrm{H}, J=7.7), 7.90(\mathrm{~d}, 1 \mathrm{H}, J=15.5)$ $7.35(\mathrm{~d}, 1 \mathrm{H}, J=4.0), 7.20(\mathrm{~m}, 1 \mathrm{H}), 6.30(\mathrm{~m}, 1 \mathrm{H}), 4.42(\mathrm{~s}, 3 \mathrm{H}), 3.88(\mathrm{~s}, 3 \mathrm{H})$.

1-(N-Methylquinol-4-yl)-2-(5-formyl- $N$-methylpyrrol-2-yl)ethene triflate (6). Freshly distilled $\mathrm{POCl}_{3}(0.711 \mathrm{~g}, 4.64 \mathrm{mmol})$ was added dropwise to anhyd DMF $(0.339 \mathrm{~g}, 4.64 \mathrm{mmol})$ 
under a nitrogen atmosphere at $-15^{\circ} \mathrm{C}$. Anhyd acetonitrile $(4 \mathrm{~mL})$ and then a solution of $5(0.918 \mathrm{~g}$, $2.32 \mathrm{mmol})$ in anhyd acetonitrile $(15 \mathrm{~mL})$ was added dropwise to the reaction mixture at $-15^{\circ} \mathrm{C}$. The mixture was stirred for $6 \mathrm{~h}$ at room temperature, the precipitate was collected to give the as a red fluorescent solid (0.530 g, $1.25 \mathrm{mmol}, 54 \%)$ : mp 234-235 ${ }^{\circ} \mathrm{C}(\mathrm{EtOH}) ;{ }^{1} \mathrm{H}$ NMR (DMSO- $\left.d_{6}\right)$ $9.67(\mathrm{~s}, 1 \mathrm{H}), 9.37(\mathrm{~d}, 1 \mathrm{H}, J=6.3), 9.03(\mathrm{~d}, 1 \mathrm{H}, J=8.5), 8.68(\mathrm{~d}, 1 \mathrm{H}, J=6.5), 8.46(\mathrm{~d}, 1 \mathrm{H}, J=8.6)$, $8.41(\mathrm{~d}, 1 \mathrm{H}, J=15.5), 8.28(\mathrm{t}, 1 \mathrm{H}, J=7.8), 8.19(\mathrm{~d}, 1 \mathrm{H}, J=15.5), 8.06(\mathrm{t}, 1 \mathrm{H}, J=7.6), 7.45(\mathrm{~d}, 1 \mathrm{H}$, $J=4.3), 7.20(\mathrm{~d}, 1 \mathrm{H}, J=4.3), 4.57(\mathrm{~s}, 3 \mathrm{H}), 4.13(\mathrm{~s}, 3 \mathrm{H})$.

\section{$N$-Methyl-2-[1-(N-methylquinol-4-yl)ethen-2-yl]-5-[1-(bis-2-benzothiazolylmethyl)}

ethen-2-yl]pyrrole triflate (2). A solution of $6(0.118 \mathrm{~g}, 0.27 \mathrm{mmol})$ in $\mathrm{EtOH}(10 \mathrm{~mL})$ was added to a solution of bis-2-benzotiazolilmethane $\mathrm{e}^{4}(0.077 \mathrm{~g}, 0.27 \mathrm{mmol})$ and piperidine $(0.1 \mathrm{~mL})$ in EtOH $(8 \mathrm{~mL})$. The reaction mixture was refluxed for $30 \mathrm{~min}$ and then cooled to room temperature. The bright violet precipitate was collected and washed with ethanol $(3 \mathrm{~mL})$ to give the product as a violet solid (0.090 g, $0.13 \mathrm{mmol}, 48.5 \%): \mathrm{mp} 220-221{ }^{\circ} \mathrm{C}(\mathrm{EtOH}) ;{ }^{1} \mathrm{H}$ NMR (DMSO- $\left.d_{6}\right) \quad 9.22(\mathrm{~d}, 1 \mathrm{H}, J$ $=6.7), 8.90(\mathrm{~d}, 1 \mathrm{H}, J=8.5), 8.61(\mathrm{~d}, 1 \mathrm{H}, J=6.6), 8.37(\mathrm{~d}, 1 \mathrm{H}, J=8.8), 8.26(\mathrm{~d}, 1 \mathrm{H}, J=7.5), 8.25$ $(\mathrm{d}, 1 \mathrm{H}, J=15.5), 8.21(\mathrm{~d}, 1 \mathrm{H}, J=7.7), 8.20(\mathrm{t}, 1 \mathrm{H}, J=7.6), 8.15(\mathrm{~s}, 1 \mathrm{H}), 8.08(\mathrm{~d}, 1 \mathrm{H}, J=7.9), 8.04$ $(\mathrm{d}, 1 \mathrm{H}, J=15.3), 7.99(\mathrm{~d}, 1 \mathrm{H}, J=8.1), 7.98(\mathrm{t}, 1 \mathrm{H}, J=7.6), 7.67(\mathrm{t}, 1 \mathrm{H}, J=7.2), 7.61(\mathrm{t}, 1 \mathrm{H}, J=$ 7.3), $7.54(\mathrm{t}, 1 \mathrm{H}, J=7.7), 7.64(\mathrm{t}, 1 \mathrm{H}, J=7.7), 7.28(\mathrm{~d}, 1 \mathrm{H}, J=4.5), 5.71(\mathrm{~d}, 1 \mathrm{H}, J=4.4), 4.48(\mathrm{~s}$, $3 \mathrm{H}), 4.08$ (s, 3H); ${ }^{13} \mathrm{C}$ NMR (DMSO- $\left.d_{6}\right) 166.88,163.93,153.25,152.90,151.96,147.00,138.80$, $136.02,135.71,134.73,134.67,133.20,128.90,126.87,126.72,126.30,126.06,125.88,125.50$, $124.92,124.46,123.51,122.75,122.64,122.19,119.23,117.69,115.87,115.12,114.53,45.03$, 31.11. Anal. Calcd for $\mathrm{C}_{34} \mathrm{H}_{25} \mathrm{~F}_{3} \mathrm{~N}_{4} \mathrm{O}_{3} \mathrm{~S}_{3}: \mathrm{C}, 59.12 ; \mathrm{H}, 3.65 ; \mathrm{N}, 8.11$. Found: $\mathrm{C}, 60.06 ; \mathrm{H}, 3.28 ; \mathrm{N}$, 8.46. 


\section{References.}

1. Abbotto, A.; Alanzo, V.; Bradamante, S.; Pagani, G. A. J. Chem. Soc., Perkin Trans. 2 1991, 481.

2. Bradamante, S.; Facchetti, A.; Pagani, G. A. J. Phys. Org. Chem. 1997, 10, 514.

3. Zhu, D.; Kochi, Jay K. Organometallics 1999, 18, 161.

4. Rai, C.; Braunwarth, J. B. J. Org. Chem. 1961, 26, 3434. 